\title{
Observational Model
}

National Cancer Institute

\section{Source}

National Cancer Institute. Observational Model. NCI Thesaurus. Code C126064.

The trial design developed to compare biomedical and/or health outcomes in pre-defined and non-assigned groups of individuals. 